\title{
Performance Enhancement of Raman-based Distributed Temperature Sensors using Simplex Codes
}

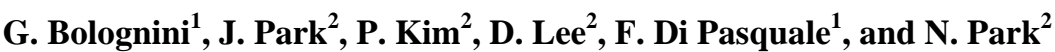 \\ 1: Scuola Superiore Sant'Anna, Piazza Martiri della Libertà 33, 56127 Pisa, Italy. E-mail: gabriele.bolognini@cnit.it \\ 2: School of EECS, Seoul National University, Korea.E-mail: nkpark@plaza.snu.ac.kr
}

\begin{abstract}
:
Using 63 bit simplex coding we demonstrate enhanced performance in Raman-based distributed temperature sensors using low-power $(80 \mathrm{~mW})$ laser diodes. Achieved $5.8 \mathrm{~dB}$ improvement in dynamic range allows for temperature sensing over $17 \mathrm{~km}$ with $15 \mathrm{~m} / 5 \mathrm{~K}$ spatial/temperature resolution.

(C) 2005 Optical Society of America

OCIS codes: $060.2370,190.5650$
\end{abstract}

\section{Introduction}

Optical fiber distributed temperature sensors (DTS) based on spontaneous Raman scattering (SRS) have been intensively studied and developed commercially during the past years [1]. In most schemes the temperature dependent backscattered SRS radiation is usually monitored using optical time-domain reflectometry (OTDR) techniques, in order to give an accurate location of temperature variations along the fiber length. With these techniques, measurements of temperature variations as small as $4 \mathrm{~K}$, with a spatial resolution of $10 \mathrm{~m}$, over $10 \mathrm{Km}$ single mode fiber have been previously reported [2], and the commercial development of DTS systems has been possible. The main drawbacks of Raman-based temperature sensors are caused by the low intensity levels of backscattered Raman light, which force one to use high sensitivity detectors and high power optical pulses in OTDR to enhance performance. Eventually, the maximum useful input power, and attainable performance, is limited by the onset on nonlinear effects (such as stimulated Raman and Brillouin scattering). The high power values generally used in ODTR pulses (of the order of $1 \mathrm{~W}$ ), along with the high sensitivity requirements at detection, have represented a limiting drawback comparing to other distributed sensors, such as systems based on spontaneous Brillouin scattering, which are characterised by higher backscattered power levels, but at the same time by more complicated detection schemes, due to the small spectral separation between pump and Brillouin wavelengths [3].

The use of simplex coding (S-coding) in conjunction with OTDR can be effectively used to enhance the signal-tonoise ratio (SNR) of the backscattered detected light without sacrificing the spatial resolution; actually, simplex codes have been demonstrated to be the most efficient among other suitable coding schemes, allowing for a good improvement in SNR even at short code lengths [4]. Experiments reported $9 \mathrm{~dB}$ of coding gain [5], achieved with simplex coding with respect to conventional OTDR. The same coding technique can also be effectively used in DTS systems, where the SNR improvement can in principle be exploited to increase the measurement distance, to obtain a more accurate spatial resolution or to reduce the input pulse power level.

In this paper, for the first time to our knowledge, coding techniques (namely simplex coding with 63 bit code length) have been used in Raman-based DTS. This has led to $5.8 \mathrm{~dB}$ dynamic range enhancement, allowing for the novel use of low power ( $80 \mathrm{~mW})$, commercially available pump sources in Raman-based distributed sensing.

\section{Theory}

Monitoring the backward propagating Anti-Stokes and Stokes lines in an OTDR system allows for distributed temperature sensing, since their intensities are dependent on the fiber temperature, due to the change in phonon distribution. In practice, the ratios of Anti-Stokes to Stokes light intensities or Anti-Stokes to backscattered pump light intensities are typically used for distributed temperature measurement, since they are not only dependent on the absolute temperature according to simple analytical relations [6], but also because they are independent on splice, bend or connector losses. In particular, the ratio between the backward propagating Anti-Stokes intensity $I_{A S}$ and the backscattered pump intensity $I_{B S}$, which was used in our measurements, is approximately proportional to:

$$
\frac{I_{A S}}{I_{B S}} \propto\left[\exp \left(\frac{h \Delta v_{R}}{k T}\right)-1\right]^{-1}
$$

where $h$ in the Planck constant, $k$ is the Boltzmann constant, $T$ is the absolute temperature, and $\Delta v_{R}$ is the separation between Raman Anti-Stokes and pump light frequencies. 


\section{OTuL1.pdf}

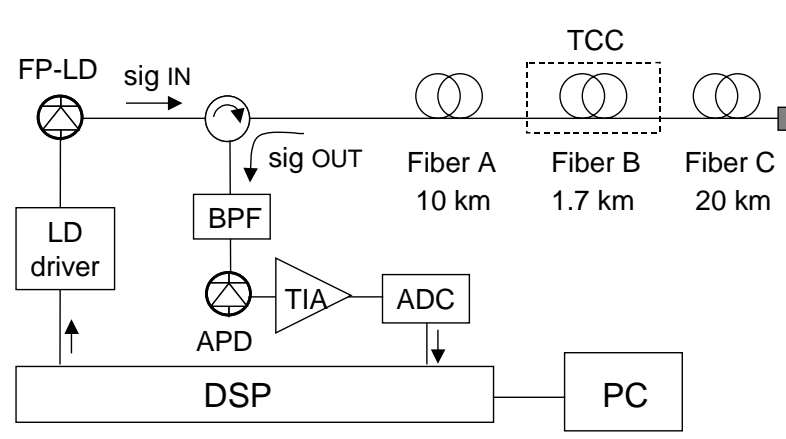

(a)

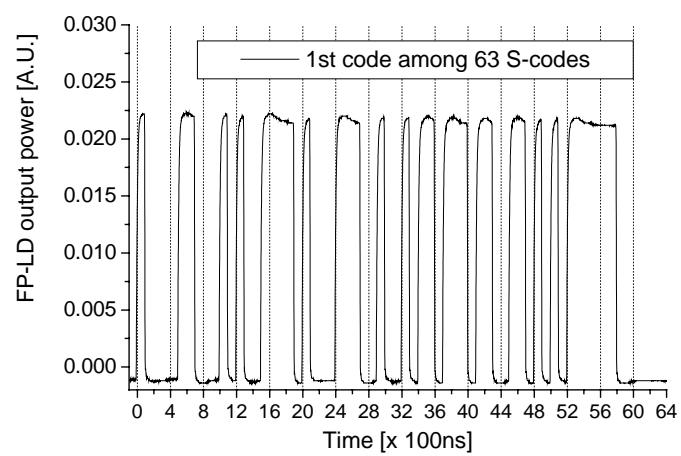

(b)

Fig.1. (a) Experimental set-up. (b) Output power of FP-LD modulated according to $1^{\text {st }}$ codeword of 63 code set.

Equation (1), properly corrected to take into account for the different attenuation experienced along the optical path at backscattered pump and Anti-Stokes wavelengths, was used in our experiments to measure the temperature variation along the optical fiber, with respect to the known absolute temperature (300 K) at the fiber input.

Coding techniques in OTDR schemes provide substantial SNR improvements, which can be quantified by the coding gain, defined as the ratio of SNR obtained with coded OTDR to SNR obtained with conventional ODTR at a given distance, and equal total number of measured traces. For simplex coding, the coding gain $G_{c o d}$ for an arbitrary code length $L$ is given by [5]:

$G_{C O D}=\frac{L+1}{2 \sqrt{L}}$.

\section{Experiment}

The implemented DTS system is reported in Fig. 1(a). To experimentally verify the proposed technique, we used an in-house PC-controlled OTDR board employing a digital signal processor (DSP), capable of modulating the light source through a laser driver with pulse patterns according to S-codes, as well as of working in single pulse operation (conventional OTDR) [6].

The light source is provided by a Fabry-Perot laser diode (FP-LD) with $80 \mathrm{~mW}$ peak power, $1550 \mathrm{~nm}$ center wavelength, and $10 \mathrm{~nm}$ FWHM spectral width, thus preventing coherent effects. The FP-LD is directly modulated according to simplex coding, with 100 ns single bit pulsewidth. The input pulsed light is coupled to the sensing fiber through an optical circulator, and the backscattered optical signals from the sensing fiber are redirected to a large bandwidth optical band-pass filter (BPF), which allows one to select the Anti-Stokes light at $1450 \mathrm{~nm}$ or the backscattered pump light at 1550nm. The light power is then detected by a high sensitivity InGaAs avalanche photodiode (APD), amplified by a high gain transimpedance amplifier (TIA), with $3 \mathrm{MHz}$ bandwidth. An analog-todigital converter (ADC) was used to sample the incoming analog signal at $20 \mathrm{MHz}$, and the coded sampled traces transmitted to the PC, where the trace acquisition and the appropriate de-coding process were carried out. The achieved spatial resolution limit (defined by the 10\% to 90\% response time) with the present DTS system was measured to be $15 \mathrm{~m}$, mainly limited by the response bandwidths of the TIA and ADC.

As a sensing fiber, three connectorised spools (A, B, C) of standard single mode fiber (SSMF) have been used in the experiments (with lengths: $A=10 \mathrm{~km}, \mathrm{~B}=1.7 \mathrm{Km}, \mathrm{C}=20 \mathrm{~km}$ ), using $\mathrm{FC} / \mathrm{APC}$ connectors to avoid reflections. Temperature of spool B has been controlled using a temperature-controlled chamber (TCC) while fiber spools A and $\mathrm{C}$ have been kept at room temperature (300 K). S-coding, with 63 bit codeword length and 63 different codewords, has been used in our experiments. The FP-LD output power modulation, according to one 63 bit codeword, is shown in Fig. 1(b). In the experiments, the temperature of spool B was kept at $300 \mathrm{~K}$ and then raised to $330 \mathrm{~K}$.

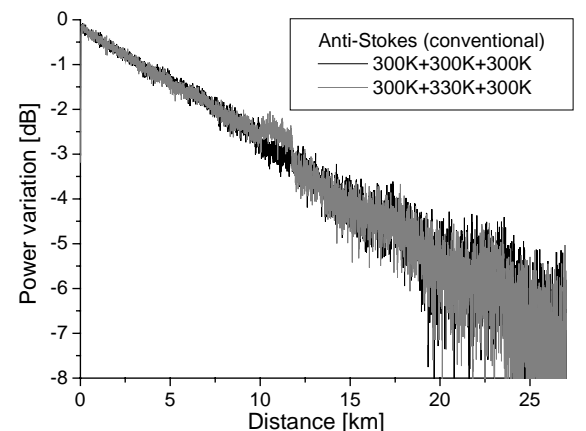

(a)

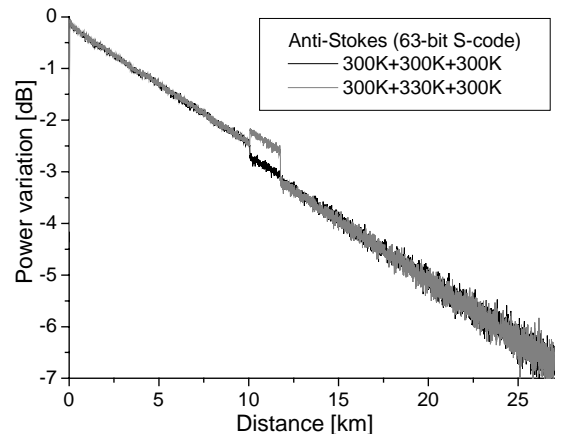

(b)

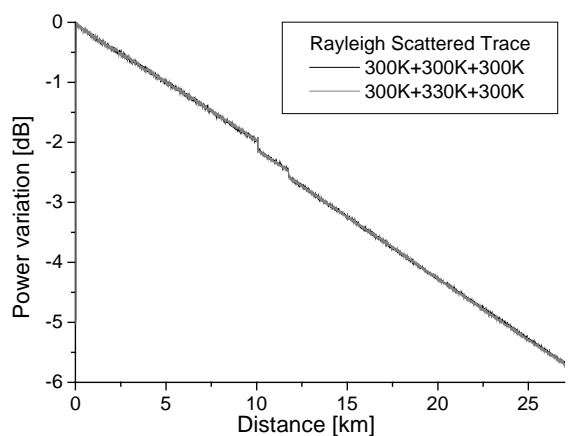

(c)

Fig. 2. Measured OTDR traces at room temperature (black line) and heated fiber B (gray line). (a) Averaged trace of Anti-Stokes power using conventional OTDR (b) De-coded trace of Anti-Stokes power using coded OTDR (c) Averaged trace of backscattered pump power with conventional OTDR. 


\section{OTuL1.pdf}

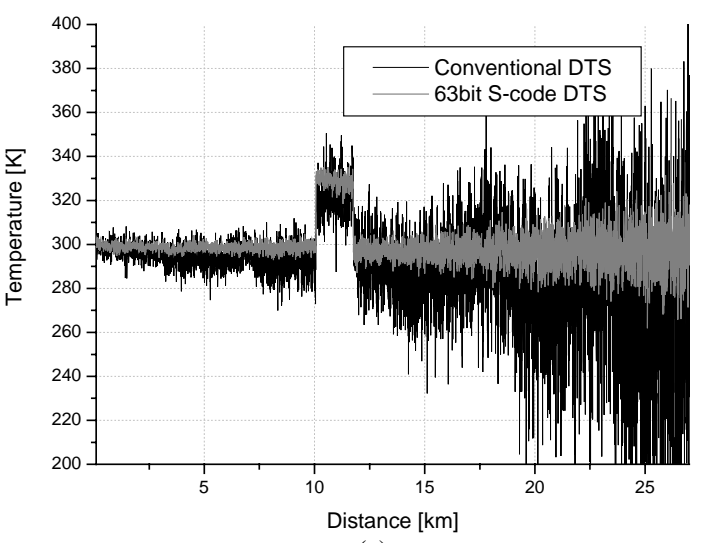

(a)

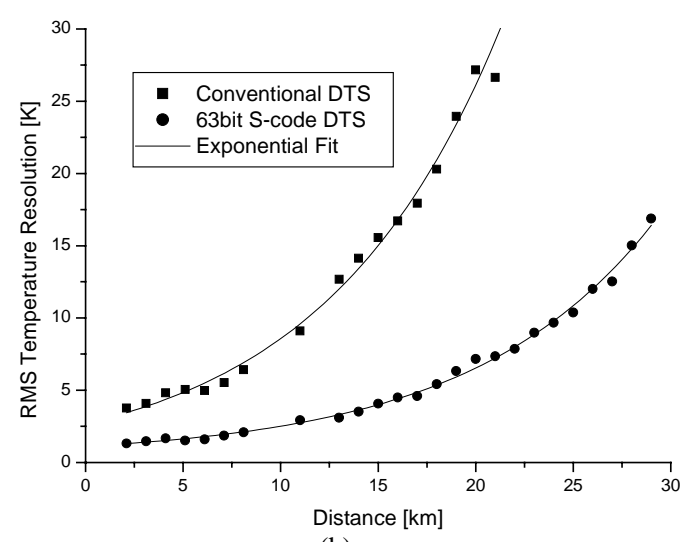

(b)

Fig. 3. (a) Temperature distribution vs distance with conventional (black line) and coded OTDR (gray line). (b) RMS temperature resolution vs distance using conventional (squares) and coded OTDR (circles), and exponential fit (solid line).

The traces for Raman Anti-Stokes line were measured by using conventional and coded OTDR, in order to quantify the SNR improvement given by coding; conversely, the trace of backscattered pump light was measured with conventional OTDR only, since the corresponding SNR values are about $30 \mathrm{~dB}$ higher than for Anti-Stokes line. For calculating averaged trace in conventional OTDR, the number of measured traces was 180000 (corresponding to about $90 \mathrm{~s}$ measurement time excluding processing overhead), while, for coded OTDR, the number of measured traces for a single 63-bit codeword was only 2857. In this way the total number of traces measured for calculating the de-coded ODTR trace is the same as conventional OTDR (63x2857), hence allowing a thorough comparison of benefits provided by coding with respect to conventional OTDR.

Fig. 2 reports the experimental OTDR traces (one-way backscattered power versus fiber length) obtained with the sensing fiber kept at room temperature (300 K, black line), and with heated spool B (330 K, gray line). Fig. 2(a) and Fig. 2(b) show the averaged OTDR traces of the backward-propagating Anti-Stokes light, respectively obtained with conventional ODTR and 63-bit simplex coding. The increase in Anti-Stokes intensity in the heated fiber section is evident from both graphs; the benefits provided by 63-bit coding with respect to conventional OTDR in terms of noise reduction (with same averaging) are also clearly visible from the graphs. Fig. 2(c), conversely, shows the averaged traces of the temperature independent backscattered pump light, obtained with conventional OTDR only (the curves are superimposed in this case). In order to quantify the benefits provided by simplex coding, the ratio $I_{A S} / I_{B S}$, corrected for wavelength dependent losses, has been used for temperature assessing.

Result is shown in Fig. 3(a), where the calculated temperature is reported versus fiber length, in case of conventional OTDR (black line) and using 63 bit coding (gray line); benefits of coding are evident, providing improved temperature resolution with respect to conventional ODTR at every distance value. Achieved temperature resolution has also been calculated, as root mean square (RMS), in case of conventional and coded OTDR.

Results of calculated temperature resolution versus distance are shown in Fig. 3(b). In case of conventional OTDR, achieved temperature resolution is better than $5 \mathrm{~K}$ only for distances smaller than $4 \mathrm{Km}$. Using coded OTDR in the same conditions permits to obtain, for example, a temperature resolution better than $5 \mathrm{~K}$ for distances up to about $17.5 \mathrm{Km}$, hence providing $12.5 \mathrm{Km}$ distance enhancement. At Anti-Stoke wavelength, this reflects into an improvement in dynamic range of about $5.8 \mathrm{~dB}$, which is well in agreement with theory, predicting a dynamic range enhancement of $6 \mathrm{~dB}$ with this coding length, as from Eq. (2). Moreover, further improvements in dynamic range and coding gain could in principle be obtained by using longer code sequences, according to Eq. (2).

In conclusion, we have demonstrated for the first time the improvement of Raman-based DTS using simplex coding (with 63 bit code length). Experiments showed an enhancement of about $5.8 \mathrm{~dB}$ in dynamic-range compared with no coding, and use of coding has permitted to implement a DTS system with $5 \mathrm{~K}$ temperature resolution and $15 \mathrm{~m}$ spatial resolution over $17 \mathrm{Km}$ standard single-mode fiber, using low-power (80 mW), commercially available FabryPérot laser diodes

\section{References}

1. M. Ahangrani et al., "Spontaneous Raman scattering in optical fibers with modulated probe light for distributed temperature Raman remote sensing”, J. Lightwave Technol., 17, 1379-1391 (1999).

2. H. H. Kee et al., “1.65mm Raman-based distributed temperature sensor”, Electron. Lett., 35, 1869-1870 (1999).

3. Y. T. Cho et al., “ 50-km single-ended spontaneous-Brillouin-based distributed-temperature sensor exploiting pulsed Raman amplification”, Opt. Lett., 28, 1651 (2005).

4. M. D. Jones, “Using simplex codes to improve OTDR sensitivity”, IEEE Photon. Technol. Lett., 15, 822-824 (1993).

5. D. Lee et al, "Analysis and experimental demonstration of simplex coding technique for SNR enhancement of OTDR ," LTIMC-15, Proceedings IEEE LTIMC, New York, October 2004.

6. D. Lee et al., "SNR enhancement of OTDR using biorthogonal codes and generalised inverses”, IEEE Photon. Technol. Lett., 17, 163-165 (2005).

7. J. P. Dakin et al., "Distributed optical fiber temperature sensor using a semiconducotr light source and detector”, Electron. Lett., 21, 569570 (1985). 